\title{
On the Frequency Scaling of Rain Attenuation for Space Communications
}

\author{
Dr Boris C. Grémont ${ }^{\# 1}$ \\ ${ }^{\#}$ Electronic \& Computer Engineering Department, University of Portsmouth \\ Anglesea Road, Portsmouth, PO1 3DJ, UK \\ ${ }^{1}$ boris.gremonte port.ac.uk
}

\begin{abstract}
We present a statistical model of the scaling factor of rain attenuation encountered in dual-frequency satellite communication systems. The new model considers the impact of the random variations of rain drop size distribution on tropospheric slant path rain attenuation. The model is validated and calibrated using known empirical results.
\end{abstract}

\section{INTRODUCTION}

Accurate knowledge of the frequency scaling factor of rain attenuation is particularly useful for the design of trunk uplinks links for $\mathrm{Ku} / \mathrm{Ka} / \mathrm{V}$ band broadcast satellite systems. The Frequency Scaling Factor (FSF) of rain is critical for Up-Link Power Control (ULPC) systems for which measurements of the rain attenuation on a down-link frequency beacon are used to guess the up-link attenuation. Any model of the FSF should include a measure of the variability or spread of the FSF around its mean.

\section{New SCALING FACTOR MOdEL}

We present a model that looks at the impact of the random variations in rain drop size distribution (DSD) along the rain path, which is believed to be the main cause for the variability of the instantaneous frequency scaling factor of rain attenuation. To do so, one must first consider the specific attenuation $\alpha(R, l)$ (in $\mathrm{dB} / \mathrm{km}$ ) of rain given by [1]:

$\alpha(R, l)=4.343 \cdot 10^{-3} \int_{0}^{\infty} Q_{t}(r, \lambda, m) n(r) d r \approx a R^{b}$

$R(\mathrm{~mm} / \mathrm{h})$ denotes the rain rate, $Q_{t}(r, \lambda, m)$ (in $\left.\mathrm{m}^{2}\right)$ is the scattering cross-section of the drops having radii between $r$ and $r+d r$ (in $\mathrm{mm}$ ). It depends on drop radii, wavelength $\lambda(\mathrm{m})$ and the complex refractive index $m$ of rain. $n(r) \equiv N_{0} \cdot e^{-\Lambda \cdot r}$ is the drop-size distribution (in $\mathrm{m}^{3} \mathrm{~mm}^{-1}$ ).

The parameters $N_{0}\left(\mathrm{~m}^{3} \mathrm{~mm}^{-1}\right)$ and $\Lambda \equiv a R^{b}\left(\mathrm{~mm}^{-1}\right)$ are empirical parameters determined from experiment.

$L(\mathrm{~km})$ is the total length of the slant path through rain, the interval $[l-d l l], 0 \leq l \leq L(\mathrm{~km})$ is the small portion of slant path over which the specific attenuation is constant.
Only the DSDs proposed by Joss et al in [2] are considered. They found that the DSD varies considerably for different types of rain activity. The Joss-Drizzle distribution (J-D) is associated with very light widespread rain composed mostly of small raindrops. The Joss-Thunderstorm distribution (J-T) characterises the DSD of convective rain with high concentration of large drops. The widespread distribution ( $\mathrm{J}$ $\mathrm{W})$ is an intermediate case and can be seen as a long-term average distribution. The validity of the approximation in Eq. (1) has been demonstrated analytically by Olsen et al in [3] and has further been verified experimentally. For convenience, the values calculated by Olsen et al. are given in Table I for a rain temperature of $0^{\circ} \mathrm{C}$.

TABLE I

$a$ AND $b$ COEFFICIENTS FOR J-T AND J-D DISTRIBUTIONS

\begin{tabular}{|c|l|l|l|l|}
\hline$f(\mathrm{GHz})$ & \multicolumn{2}{|c|}{ J-T } & \multicolumn{2}{c|}{ J-D } \\
\hline 10 & 0.0169 & 1.076 & 0.0114 & 0.968 \\
\hline 11 & 0.0212 & 1.065 & 0.0141 & 0.977 \\
\hline 12 & 0.0262 & 1.052 & 0.0172 & 0.985 \\
\hline 15 & 0.0466 & 1.010 & 0.0282 & 1.003 \\
\hline 20 & 0.0983 & 0.946 & 0.0530 & 1.020 \\
\hline 25 & 0.173 & 0.884 & 0.0861 & 1.033 \\
\hline 30 & 0.274 & 0.823 & 0.1280 & 1.044 \\
\hline 35 & 0.372 & 0.783 & 0.1800 & 1.053 \\
\hline 40 & 0.451 & 0.760 & 0.2410 & 1.058 \\
\hline 50 & 0.629 & 0.709 & 0.3870 & 1.053 \\
\hline & $a_{J-T}$ & $b_{J-T}$ & $a_{J-D}$ & $b_{J-D}$ \\
\hline
\end{tabular}

In the following, the subscript or superscript $i$ equals either $u$ or $d$ depending on whether up-link frequency (e.g. $30 \mathrm{GHz}$ ) or down-link frequency (e.g. $20 \mathrm{GHz}$ ) are considered

respectively. The total attenuation $A_{i}(R)(\mathrm{dB})$ on a particular slant path of total length $\mathrm{L}$ is given by:

$$
A_{i}(R)=\int_{0}^{L} \alpha_{i}(R, l) d l \approx \bar{\alpha}_{i}(R) L\left(R, f_{i}\right) .
$$

$\bar{\alpha}_{i}(R)$ denotes the path-averaged specific attenuation and $L\left(R, f_{i}\right)$ in $\mathrm{km}$ denotes the average path length through rain cells. It takes into account, on a long-term basis, the spatial distribution of rain cells along the satellite slant-path and the variation of the $0^{\circ} \mathrm{C}$ isotherm level. The average effective path length may in general be also dependent upon frequency. 
However, it will be assumed here that this is not the case i.e. that the path length is only a function of $R$ [4][5]:

$L(R)=\left[\begin{array}{l}7.412 \cdot 10^{-3} \cdot R^{0.766}+\ldots \\ \left.\ldots+\left(0.2323-1.806 \cdot 10^{-4} \cdot R\right) \cdot \cos \theta\right)\end{array}\right]^{-1}$

From these definitions, the instantaneous FSF $k(R)$ of rain attenuation can be written:

$$
k(R)=\frac{A_{u}(R)}{A_{d}(R)}=\frac{\alpha_{u}(R)}{\alpha_{d}(R)} .
$$

The FSF is thus described as a function of the rain rate (i.e. the independent variable) does not depend on the average path length and it depends only on the path averaged specific attenuations. The variability of the FSF is now described in terms of random variations of the path-averaged specific attenuations on the up and down-link. We treat Eq. (3) as the ratio of two random variables with mean $\bar{\alpha}_{u}(R)$ and $\bar{\alpha}_{d}(R)$ and corresponding variances $\sigma_{u}^{2}(R)$ and $\sigma_{d}^{2}(R)$. It can be shown ([6]) that:

$$
\bar{k}(R) \approx \frac{\bar{\alpha}_{u}}{\bar{\alpha}_{d}}+\frac{1}{\bar{\alpha}_{d}^{2}}\left(\frac{\bar{\alpha}_{u}}{\bar{\alpha}_{d}} \sigma_{d}^{2}-r \sigma_{u} \sigma_{d}\right),
$$

and

$$
\sigma_{k}^{2}(R) \approx\left(\frac{\bar{\alpha}_{u}}{\bar{\alpha}_{d}^{2}}\right) \sigma_{d}^{2}-\frac{2 r \sigma_{u} \sigma_{d} \bar{\alpha}_{u}}{\bar{\alpha}_{d}^{3}}+\frac{\sigma_{u}^{2}}{\bar{\alpha}_{d}^{2}} .
$$

The parameter, $r$, denotes the correlation coefficient between up and down-link attenuations i.e.

$$
r=\frac{\left\langle A_{u}(R)-\bar{A}_{u}(R)\right\rangle\left\langle A_{d}(R)-\bar{A}_{d}(R)\right\rangle}{\sigma_{A_{u}} \cdot \sigma_{A_{d}}}
$$

and it is easily measurable using time series of rain attenuation. In this paper, based on our measurements, we use $r=0.92$. In order to compute Eqs. (4) and (5), we need to determine the mean and standard deviations of the up and down-link specific attenuations. Let us consider fig. 1 that shows the specific attenuations for the J-D and J-T distributions with coefficients tabulated in Table I.

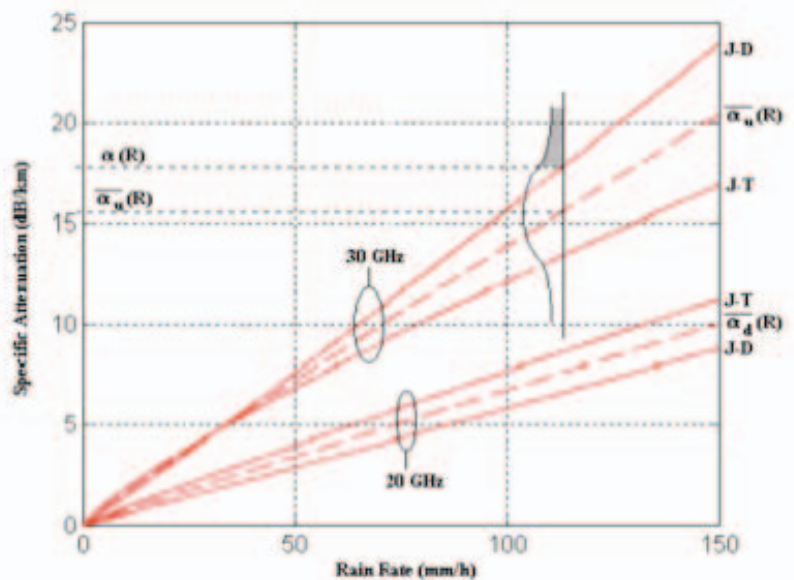

Fig. 1 Specific attenuations at $0^{\circ} \mathrm{C}$ at 20 and $30 \mathrm{GHz}$ (J-T and J-D distributions).
The average specific attenuation $\bar{\alpha}_{i}(R)$ is depicted by the dashed lines in Fig. 1 which are chosen to stand in the middle of the J-D and J-T distributions, i.e.:

$$
\bar{\alpha}_{i}(R)=0.5\left(a_{J-T}^{i} R^{b_{J-T}^{i}}+a_{J-D}^{i} R^{b_{J-D}^{i}}\right) .
$$

Things are slightly more complicated for the standard deviations $\sigma_{u}(R)$ and $\sigma_{d}(R)$.

Let us assume that the temporal variations in drop size distribution can be reasonably well modelled as conditionally Gaussian i.e. the specific attenuation given a specific rain rate, $R$, has a normal density function with conditional mean $\bar{\alpha}_{i}(R)$ and standard deviation $\sigma_{i}(R)$ given by:

$$
f\left(\alpha_{i}(R)\right)=\frac{1}{\sqrt{2 \pi} \cdot \sigma_{i}(R)} \cdot \exp \left[-\frac{\left(\alpha_{i}(R)-\bar{\alpha}_{i}(R)\right)^{2}}{2 \cdot \sigma_{i}^{2}(R)}\right]
$$

Then the probability that $\alpha_{i}(R)$ exceeds a value $\alpha(R)$ is :

$$
P\left(\alpha_{i} \geq \alpha\right)=Q\left(\frac{\left|\alpha_{i}-\bar{\alpha}_{i}\right|}{\sigma_{i}}\right) \triangleq \frac{P \%}{200},
$$

where we used the error function:

$$
Q(x)=\int_{x}^{\infty} \frac{1}{\sqrt{2 \pi}} \exp \left(\frac{-u^{2}}{2}\right) d u .
$$

Then the standard deviation can be estimated by inverting Eq. (7) giving the sought formula:

$$
\sigma_{i}(R)=\frac{\left|\alpha_{i}(R)-\bar{\alpha}_{i}(R)\right|}{Q^{-1}\left(\frac{P \%}{200}\right)}
$$

where $Q^{-1}(Q(x))=x$.

The quantity $P \%$ is introduced to denote the time percentage for which the specific attenuation $\alpha_{i}(R)$ lies outside the interval $\alpha_{i}(R) \geq\left|\alpha_{J-T}(R)-\alpha_{J-D}(R)\right|$ and it corresponds to twice the shaded are shown in Fig. 1. The value $P \%$ (corresponding to twice the shaded area in Fig. 1) is a measure of the adequacy of the J-T and J-D at representing the 'extremes' in rain drop size regimes over a long-term period. Note the absolute value is introduced in Eq. (5) as a way to ensure that $\sigma_{i}(R) \geq 0$. Without the absolute value, the standard deviation of the $30 \mathrm{GHz}$ specific attenuation would be negative, as shown in Fig. 1 for $R>30 \mathrm{~mm} / \mathrm{h}$. This rather peculiar situation is principally due the exponents $b_{\mathrm{JT}}$ and $b_{\mathrm{JD}}$ in Table I, which make the JT specific attenuation smaller than the JD specific attenuation for $\mathrm{R}>30 \mathrm{~mm} / \mathrm{h}$.

\section{MOdEL CALIBRATION \& VALIDATION}

It is useful to plot $k(R)$ and then changing the $R$ axis into the down link attenuation $\mathrm{A}_{\mathrm{d}}$ axis using the one-to-one mapping $A_{d}=A_{d}(R)=a_{d} \cdot R^{b_{d}} \cdot L(R)$. A remark at this stage is that the FSF (its mean and/or variance) depends crucially on the average length $L(\mathrm{~km})$ of the slant path through rain cells. The sensitivity of the mean FSF on the 
correlation factor $r$ has been investigated in the range $0.90<r<1$. It was found that $r$ does not affect the mean FSF significantly. The model parameter $P \%$, however, has a small but noticeable impact on $\bar{k}(R)$ for levels of down-link attenuation around $10 \mathrm{~dB}$ as shown in Fig. 2 for the 30/20 $\mathrm{GHz}$ frequency pair at an elevation angle of 30 degrees.

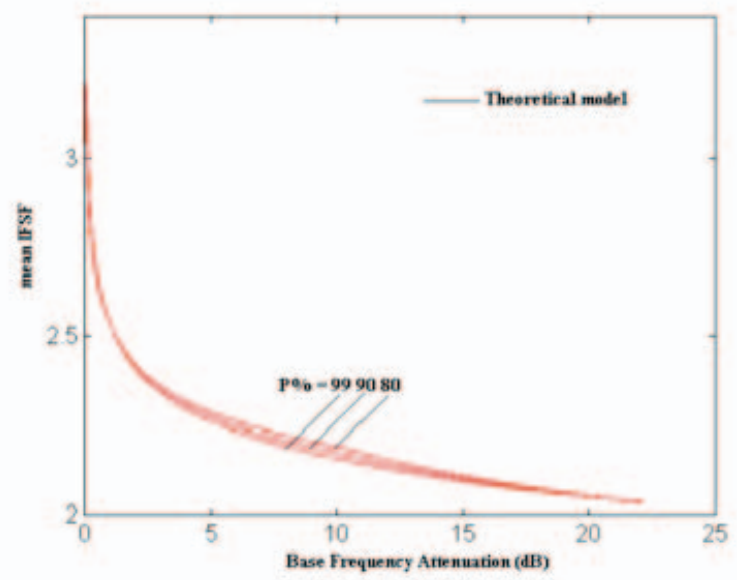

Fig. 2 Variation of the mean FSF $\bar{k}$ of rain attenuation as a function of base downlink attenuation $A_{d}$ for different values of $\mathrm{P} \%$.

A similar analysis has been applied to the standard deviation of the FSF. This is shown in Fig. 3. Quite logically, lower correlation factors yielded slightly higher standard deviations. However, as expected, $\sigma_{k}(R)$ is more importantly affected by the value of the model parameter $P \%$, which thus should be evaluated accurately later in this paper. Results compiled by Mauri, Paraboni and Riva in [7] are also displayed in Fig. 3. The authors analysed the FSF of rain attenuation for the $30 / 20 \mathrm{GHz}$ pair. They only studied 14 rain events collected during the Olympus experiment at the site of Spino d'Adda. The authors provided a table of empirical values standard deviation of the FSF for base attenuation levels between 1.5 and $22.5 \mathrm{~dB}$ in steps of $1 \mathrm{~dB}$. These empirical values, taken directly from Table 4 of [7] are shown by circles in Fig. 3. The fit between the theoretical and the practical results of Spino d'Adda is good for values of downlink attenuation levels between 5 and $10 \mathrm{~dB}$ for $P \%=90 \%$.

For levels above $10 \mathrm{~dB}$, both theory and practice predict that the standard deviation should decrease, although the Spino d'Adda data shows a sharper decrease than the theory. For levels of attenuation below $5 \mathrm{~dB}$, theory and practice predict similar trends although the horizontal scaling is not the same. In particular, both theory and practice show that there should be a minimum standard deviation between 1 and $5 \mathrm{~dB}$. Both theory and practice also show a marked change in standard deviation for a base frequency attenuation of $10 \mathrm{~dB}$. This very peculiar behaviour clearly demonstrates that the proposed model in this paper is definitely on the right track although there are some differences. As discussed before, this change in characteristics is due to the fact that the J-D specific attenuation becomes less than the J-T specific attenuation (Fig. 1) at $30 \mathrm{GHz}$ and rain rate of $30 \mathrm{~mm} / \mathrm{h}$. This suggests that the
$\mathrm{J}-\mathrm{T}$ and J-D dropsize distributions, used as an input to the model, are too approximate for our purpose. The evaluation of more adequate drop size distributions would require the computation of the scattering cross section of realistically distorted water rain drops as well as comparison with experimental beacon data. This is beyond the scope of this paper.

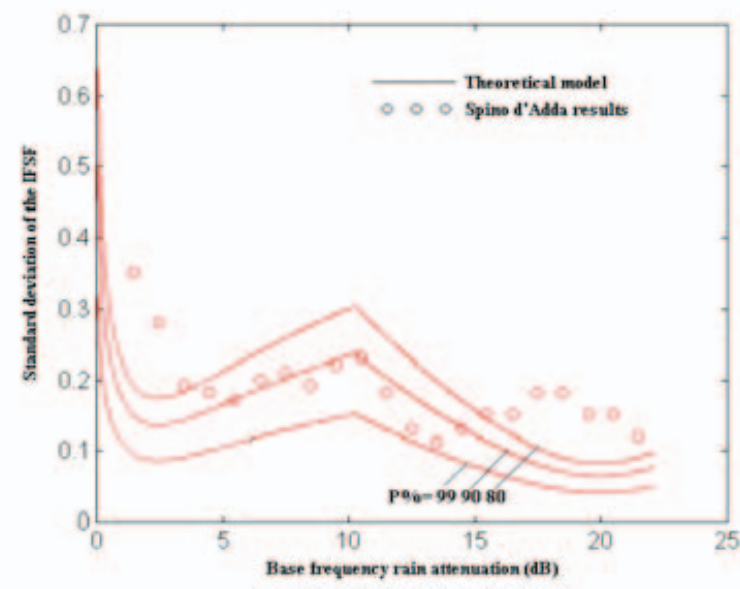

Fig. 3 Impact of the parameter $P \%$ on the standard deviation $\sigma_{k}(R)$ of the FSF of rain attenuation.

Comprehensive results were published by Rücker in [8]. The author carried out an analysis of the FSF of rain attenuation based on data collected during the Olympus experiment over a period of 2 years and 8 months for the $20 / 30 \mathrm{GHz}$ pair. Rücker's experimental results showed that the conditional cumulative distribution function of the FSF is well approximated as Gaussian for the percentiles lying between $5 \%$ and $95 \%$ and base frequency attenuation in the range 3.5 to $9.5 \mathrm{~dB}$. In view of the model proposed in the previous section, and Rücker's experimental results, the most logical model for $k$ is a conditionally normal model i.e.

$$
f(k \mid R)=\frac{1}{\sigma_{k}(R) \sqrt{2 \pi}} \exp \left[-\frac{(k-\bar{k}(R))^{2}}{2 \sigma_{k}^{2}(R)}\right]
$$

where in our case in this paper the mean $\bar{k}(R)$ and standard deviation $\sigma_{k}(R)$ can be computed using Eqs. (4) and (5).

The scaling factor can be used to estimate the up-link attenuation $A_{u}(R)$ using $A_{u}(R)=k(R) A_{d}(R)$. The probability of $A_{u}(R)$ exceeds a value $\alpha$ is given by:

$$
P_{\alpha} \triangleq \operatorname{Pr}\left\{A_{u}(R) \geq \alpha\right\}=1-Q\left(\frac{\alpha-\bar{k}(R)}{\sigma_{k}(R)}\right)
$$

Inverting this and finding an expression for the attenuation $\underline{n o t}$ exceeded with probability $P_{\alpha}$ is:

$$
\alpha=\bar{k}(R)+\sigma_{k}(R) \times Q^{-1}\left(1-P_{\alpha}\right) \mathrm{dB}
$$




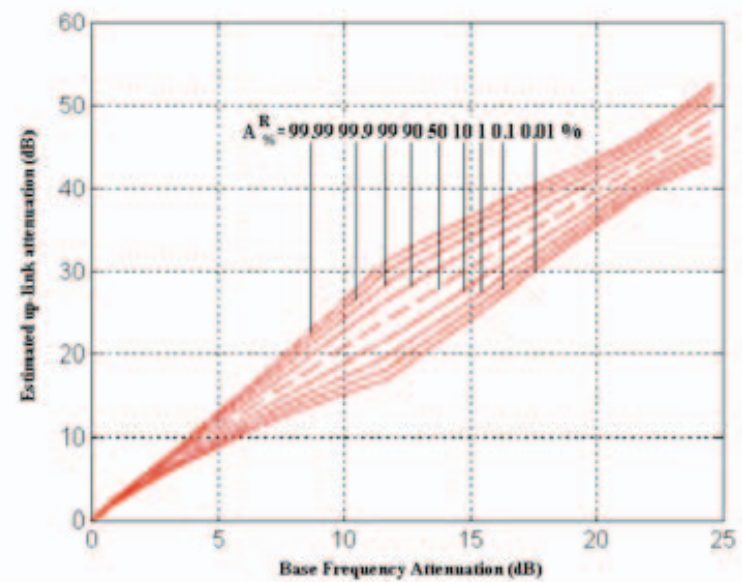

Fig. 4 Estimated up-link attenuation $\alpha$ not exceeded for $P_{\alpha} \%$ in the range $0.01 \%$ to $99.99 \%(\mathrm{P} \%=97.8 \%, 20 / 30 \mathrm{GHz}$ frequency pair).

It is important to tune the prediction of the proposed model of the FSF so that it can match published empirical results. More particularly, an appropriate value of the model parameter, $P \%$, must be estimated. Although $P \%$ can be expected to be dependent upon the climatic region of each of the links considered, the data on the IFSF of rain attenuation is still too scarce to identify such a geographical dependency. Therefore only a mean value of $P \%$ will be evaluated.

Laster and Stutzman in [9] proposed three empirical formulae for the FSF at availabilities of $P_{\alpha}=99 \%$ for the $20 / 30,12 / 20$ and 12/30 frequency pairs. These formulae are based on a comprehensive analysis of one year of data measured during the Olympus experiment over a satellite link at $13.93^{\circ}$ elevation angle. It is to date the most comprehensive study of the FSF of rain attenuation. They proposed for the following detection rules for a $99 \%$ availability:

$$
\begin{aligned}
& \hat{A}_{30}^{99 \%}=2.75 \cdot A_{20}-0.02 \cdot\left(A_{20}\right)^{2}, 1 \leq A_{20} \leq 14 \mathrm{~dB} \\
& \hat{A}_{20}^{99 \%}=2.75 \cdot A_{12}-0.02 \cdot\left(A_{12}\right)^{2}, 1 \leq A_{12} \leq 10 \mathrm{~dB} \\
& \hat{A}_{30}^{99 \%}=9.34 \cdot A_{20}-0.39 \cdot\left(A_{20}\right)^{2}, 1 \leq A_{20} \leq 4 \mathrm{~dB} .
\end{aligned}
$$

Rücker also provided a curve (Figure (3b) of [6.7]) for the $99 \%$ percentile. Polynomial curve fitting to his graph yielded the following formulas:

$$
\begin{aligned}
& \hat{A}_{30}^{99 \%}=-1.4156+2.69 \cdot A_{20}-0.0098 \cdot\left(A_{20}\right)^{2} \\
& 1 \leq A_{20} \leq 10 \mathrm{~dB} .
\end{aligned}
$$

These empirical fits were used to calibrate the proposed model of the FSF of rain attenuation. More particularly, appropriate elevation angle, frequencies and values of $P \%$ between $80 \%$ and $99.9 \%$ (in steps of $0.1 \%$ ) were fed into the model. The best value of $P \%$ was then selected when the rms error between each of the equations above and the model in section II is minimised. Correlation $r=0.93$ was assumed throughout and we used $Q^{-1}(1-0.99)=2.33$ corresponding to $99 \%$ availability. The results are given in Table II. If we take the average of the 4 identified values of $P \%$ in Table 6.3 , we get an estimate for $P \%$ of $97.8 \%$. Table II also shows that the individual values of $P \%$ are quite homogeneous and very close to $100 \%$. Therefore, the J-D and $\mathrm{J}-\mathrm{T}$ drop size distributions do indeed represent extreme rain activities.

TABLE II

IDENTIFICATION OF P\% BASED ON EXPERIMENTAL RESULTS

\begin{tabular}{|l|l|l|l|}
\hline $\begin{array}{l}\text { Frequencies } \\
\text { GHz }\end{array}$ & $\begin{array}{l}\text { Elevation } \\
\text { angle }\end{array}$ & $\begin{array}{l}\text { Optimum } \\
\text { P\% }\end{array}$ & Source \\
\hline $29.65 / 19.77$ & 13.93 & 99.7 & {$[6.8]$} \\
\hline $29.65 / 12.5$ & 13.93 & 96.9 & {$[6.8]$} \\
\hline $19.77 / 12.5$ & 13.93 & 98.2 & {$[6.8]$} \\
\hline $29.65 / 19.77$ & 30.00 & 96.4 & {$[6.8]$} \\
\hline
\end{tabular}

\section{CONCLUSIONS}

A new model of the frequency scaling factor of rain attenuation was presented in this paper. It considers the impact of random variations in drop size distribution on the mean and standard deviation of the scaling factor. This model assumes a conditionally Gaussian model of the specific attenuation conditioned on rain rate. It was assumed that most variations lie between the J-T and J-D distributions, which were used as reference or extremes in the model. The model was then validated and calibrated using empirical results published in the literature. The model is the only one of its kind since it not only permits theoretical estimation of the mean scaling factor but also the standard deviation of the scaling factor.

\section{ACKNOWLEDGMENT}

This work is associated with the research activities of European COST Action IC0802: "Propagation tools and data for integrated Telecommunication, Navigation and Earth Observation systems". For more details see: w3.cost.esf.org/index.php?id=177\&action_number=IC0802

\section{REFERENCES}

[1] L.J. Ippolito, "Radiowave propagation in satellite communications", Van Nostrand Reinhold, 1986.

[2] J. Joss, J.C. Thams, A. Waldvogel, "The variation of rain drop-size distributions at Locarno", Proc. Int. Conf. Cloud Physics, 1968, pp 369-373.

[3] R. Olsen, D.V. Rogers, D.B. Hodge, "The $a R^{b}$ relation in the calculation of rain attenuation", IEEE Trans. on Ant. and Prop., Vol. AP-26, No 2, March 1978, pp 318-329.

[4] I.P. Shkarofski, "Dependence of rain attenuation and cross-polarization on drop size distribution", IEEE Trans. on Ant. and Prop., Vol. AP-27, No 4, July 1979, pp 538-542.

[5] ESA, Second workshop of the Olympus experimenters, Volume 1, Reference book on attenuation measurement and prediction, Noordwijk, 8-10 Nov. 1994, Chapter 7: Characteristics of events, ESA ESTEC WPP-083, Noordwijk, The Netherlands, pp 87-123.

[6] A. Papoulis, S. Unnikrishhna Pillai, "Probability, random variables and Stochastic Processes", $4^{\text {th }}$ edition, McGraw-Hill, 2002, pp. 217.

[7] M. Mauri, A. Paraboni, C. Riva, "Instantaneous Frequency Scaling", OPEX $2^{\text {nd }}$ Workshop of the Olympus Experimenters, Vol. 6: Proceedings, ESA WPP-083, Noordjwick, 8-10 Nov. 1994.

[8] F. Rücker, "The impact of the variation of instantaneous frequency scaling factors on up-link power control", $A E \ddot{U}$, Vol. 48, No2, 1994, pp 119-121.

[9] J.D. Laster, W.L. Stutzman, "Frequency scaling of rain attenuation for satellite Communications links", IEEE Trans. on Ant. and Prop., Vol. AP-43, No. 11, Nov. 1995, pp 1207-1215. 